\title{
Enhanced monopole and dipole transitions in nuclei induced by $\alpha$ cluster structures
}

\author{
Makoto Ito ${ }^{1, *}$, Makoto Nakao ${ }^{1}$, and Tomoyuki Okuno ${ }^{1}$ Syuuichiro Ebata ${ }^{2}$ \\ ${ }^{1}$ Department of Pure and Applied Physics, Kansai University, Yamatecho, 3-3-35, Suita 564-8680, Japan \\ ${ }^{2}$ Laboratory for Advanced Nuclear Energy, Institute of Innovative Research, Tokyo Institute of Technology, 2-12-1-N1-16 Ookayama, \\ Meguro-ku 152-8550, Japan
}

\begin{abstract}
Enhancement of the monopole and dipole transitions in the low-lying state is discussed on the basis of the microscopic and macroscopic $\alpha$ cluster models. Theoretical calculation clearly demonstrates that the strength of the monopole and dipole transitions are strongly enhanced by the excitation in the relative motion of the $\alpha$ cluster and the residual nucleus. The transition strength induced by the $\alpha$ excitation appears as the discrete distribution, and its excitation energy is much lower than the excitation energy of the single nucleon excitation, which is expected from the naive mean field picture.
\end{abstract}

\section{Introduction}

Basic properties of ground state in nuclei can be described by mean field picture, in which individual nucleons perform single particle motions in a self-consistent mean field [1]. Coherent excitations of such the single particle motion generate various collective excitations of the nuclei [1]. On the contrary, the so-called $\alpha$ cluster structures are known to be realized in the excited states of the nuclei [2]. The $\alpha$ particle is quite stable and inert and hence, it becomes a subunit, which is a building block in constructing the intrinsic structures of the nuclear systems. The typical examples of the $\alpha$ cluster state are ${ }^{8} \mathrm{Be}=2 \alpha,{ }^{12} \mathrm{C}=3 \alpha,{ }^{16} \mathrm{O}$ $=\alpha+{ }^{12} \mathrm{C}$ and ${ }^{20} \mathrm{Ne}=\alpha+{ }^{16} \mathrm{O}$ in the lighter mass region.

The picture of the $\alpha$ cluster structures is extended to much heavier systems beyond $A=40$, such as ${ }^{44} \mathrm{Ti}=\alpha$ $+{ }^{40} \mathrm{Ca}[2,4,5]$, neighboring nuclei of ${ }^{94} \mathrm{Mo}=\alpha+{ }^{90} \mathrm{Zr}$ $[4,6]$, and ${ }^{212} \mathrm{Po}=\alpha+{ }^{208} \mathrm{~Pb}[4,7]$. In the studies of the heavy systems, the macroscopic $\alpha$ cluster model, in which the local potential for the system of $\alpha$-residual nucleus is an initial ingredient, are mainly applied. Amongst, the ${ }^{44} \mathrm{Ti}$ nucleus is the most deeply analyzed not only by the local potential approaches $[3,4]$ but also by the full microscopic approaches [5].

The $\alpha$ cluster structure mainly appears as the first excited $0^{+}$state or the yrast $1^{-}$state. The latter state corresponds to the negative parity partner of the parity doublet with the ground $0^{+}$state. Recently, the isoscalar (or electric) monopole and dipole transitions have been proposed as effective probes to identify the cluster excitation $[8,9]$. The monopole transition is strongly increased for the radial excitation in the relative wave function of the $\alpha$ cluster and a resitudal nucleus, while the dipole operator strongly responds to the dipole excitation in asymmetric

*e-mail: itomk@kansai-u.ac.jp cluster systems with an $\alpha$ particle plus a heavy core. When the $\alpha$ cluster structures are well developed, the excited $0^{+}$ state and the yrast $1^{-}$state appear at much lower excitation energy than the excitation energy based on an independent nucleon motion in a mean field picture. Therefore, the low-lying enhancement in the monopole and dipole transition is considered to be a clear sign of the excitation of $\alpha$ cluster degrees of freedom.

In this report, we show our analyses for the light nuclei, such as ${ }^{12} \mathrm{Be}[10]$ and ${ }^{18} \mathrm{O}$ [11], which are based on the microscopic and semi-microscrpic $\alpha$ cluster models, and point out that the strong monopole transition is induced by the $\alpha$ cluster excitation. Furthermore, we analyze the monopole and dipole transitions in the heavier systems, ${ }^{44} \mathrm{Ti}$ and ${ }^{104-110} \mathrm{Te}$, by applying the macroscopic $\alpha$ cluster model, which is based on the double folding potential of the $\alpha$ particle and residual core nuclei. From the calculation of the macroscopic $\alpha$ cluster model, we will demonstrate that the monopole and dipole transition is prominently enhanced at anomalously low excitation energy.

\section{Theoretical framework}

In this section, we briefly explain theoretical framework for the calculation of the light and heavy mass systems. The (semi) microscopic cluster model is applied to the light systems, while we use the macroscopic $\alpha$ cluster model in the calculation of the heavy systems.

\subsection{Micorscopic and semi-microscopic cluster models for ${ }^{12} \mathrm{Be}$ and ${ }^{18} \mathrm{O}$}

In the structure calculations of the ${ }^{12} \mathrm{Be}$ and ${ }^{18} \mathrm{O}$ nuclei, we apply the microscopic and semi-microscopic $\alpha$ cluster models, respectively, in which the anti-symmetrization effect among the nucleons are taken into account. As for 
${ }^{12} \mathrm{Be}$, we assume the six-body systems of $\alpha+\alpha+4 N$ and employ the generalized two-center cluster model (GTCM) [10], which is possible to handle the atomic structure with the binary He-clusters and the covalent structure of excess four neutrons around two $\alpha$ cores. On the contrary, the orthogonality condition model (OCM) with the $\alpha+{ }^{14} \mathrm{C}$ cluster configuration is used for the structure calculation of ${ }^{18} \mathrm{O}$ [11]. Both calculations nicely reproduce the level sequence of the $0^{+}$states. The details of these cluster models are explained in Refs.[10] and [11].

\subsection{Macroscopic $\alpha$ cluster model in ${ }^{44} \mathrm{Ti}$ and Te isotopes}

In the heavy systems, the explicit treatment of the antisymmetrization among the nucleon becomes complicated. Thus, we employ the macroscopic $\alpha$ cluster model for ${ }^{44} \mathrm{Ti}$ with $\alpha+{ }^{40} \mathrm{Ca}$ and ${ }^{104-110} \mathrm{Te}$ with $\alpha+{ }^{100-106} \mathrm{Sn}$. The basic framework is same as those shown in Refs. [3, 4]. In the macroscopic $\alpha$ cluster model, the nuclear potential of $\alpha$ and heavy cores $\left({ }^{40} \mathrm{Ca}\right.$ or $\left.\mathrm{Sn}\right)$ is calculated from the double folding (DF) model [12], which is symbolically written as a function of the $\alpha$ - core relative coordinate $\mathbf{R}$,

$$
U_{\mathrm{DF}}(\mathbf{R})=\iint \rho_{\alpha}\left(\mathbf{r}_{\alpha}\right) \rho_{C}\left(\mathbf{r}_{C}\right) \times v_{\mathrm{NN}}^{\mathrm{DDMY}}(s, \rho) \mathrm{d} \mathbf{r}_{\alpha} \mathrm{d} \mathbf{r}_{C}
$$

with $s=\left|\mathbf{r}_{C}-\mathbf{r}_{\alpha}-\mathbf{R}\right|$. Here $\mathbf{r}_{\alpha}\left(\mathbf{r}_{C}\right)$ denotes a coordinate measured from the center of mass in $\alpha$ (core nucleus). $\rho_{\alpha}\left(\mathbf{r}_{\alpha}\right)$ is the density of $\alpha$ particle, which reproduces the charge form factor, while $\rho_{C}\left(\mathbf{r}_{C}\right)$ represents the density of the core nucleus, which is calculated by the mean-filed model [13].

In Eq.(1), $v_{\mathrm{NN}}^{\mathrm{DDM} 3 \mathrm{Y}}$ represents the effective nucleonnucleon $(\mathrm{NN})$ interaction which acts between a pair of nucleons contained in the $\alpha$ particle and the core nucleus. In the present calculation, we adopt the DDM3Y (density dependent Michigan three range Yukawa) interaction [12]. The DF potential with DDM3Y is well known to reproduce the differential cross section of the $\alpha+{ }^{40} \mathrm{Ca}$ elastic scattering [3]. We calculate the energy levels with the DF potential in Eq. (1). In the calculation of the energy levels, we take into account the pseudo potential to exclude the Pauli's forbidden state and the absorbing boundary condition to handle the unbound states [14]. The Pauli's forbidden states are prepared by assuming the lowest shell model configuration for the core nucleus and considering the condition about the number of the radial nodes in the $\alpha$ - Core relative wave function.

The continuum strength function for the transition from the ground state to the unbound continua, which is induced by the external field, is calculated from the ABC calculation [14]. As for the operator of the external field, we consider the monopole $(\lambda=0)$ and dipole $(\lambda=1)$ operators, which are basically written in the nucleon degree of freedom like $[8,9]$

$$
\begin{aligned}
& \hat{O}_{\lambda=0}=\sum_{i=1}^{A \text { or } Z} r_{i}^{2} \\
& \hat{O}_{\lambda=1}=\sum_{i=1}^{A} r_{i}^{3} Y_{1,0}\left(\hat{\mathbf{r}}_{i}\right) .
\end{aligned}
$$

In Eq. (2), the summation is taken over a whole nucleons $(A)$ in the case of isoscalar monopole (IS0) transition, while it is restricted to protons $(Z)$ for the electric monopole (E0) transition. In the calculations of semimicroscopic and macroscopic $\alpha$ cluster models, we extract a part of the $\alpha$-core relative coordinate (R) from Eqs. (2) and (3) according to the definitions in Refs. [8, 9].

\section{Resuts}

\subsection{Monopole transition in ${ }^{12} \mathrm{Be}$ and ${ }^{18} \mathrm{O}$}

The results of the $0^{+}$energies and the isoscalar monopole (IS0) matrix elements for ${ }^{12} \mathrm{Be}$ are summarized in table 1. In this table, the strengths are shown in units of the single particle strength for $0 p \rightarrow 1 p$. All of the excited states, which appear below the excitation energy of $E_{x} \leq$ $15 \mathrm{MeV}$, have the strength comparable to or larger than the single particle excitation. In particular, the excitation to $0_{3}^{+}$around $E_{x} \sim 10 \mathrm{MeV}$ is the most enhanced of all the excited $0^{+}$state because this state corresponds to the direct excitation in the $\alpha-{ }^{8} \mathrm{He}$ relative motion from the ground ${ }^{12} \mathrm{Be}$ nucleus [10]. The theoretical strength of $0_{1}^{+} \rightarrow 0_{3}^{+}$ nicely reproduce the observed strength of 2.0 [15].

Table 1. $0^{+}$energies and strength of IS0 matrix elements in ${ }^{12} \mathrm{Be}$. The theoretical energies are shown in units of $\mathrm{MeV}$, while the energies in parentheses mean the experimental data. The IS0 strength of theory (Th.) and experment (Exp.) are shown in unit of the single particle strength [10]. Experimental data of IS0 is taken from Ref. [15]

\begin{tabular}{llll}
\hline State & Energy & IS0 (Th.) & IS0 (Exp.) \\
\hline $0_{2}^{+}$ & $2.2(2.3)$ & 2.6 & - \\
$0_{3}^{+}$ & $9.8(10.5)$ & 3.5 & 2.0 \\
$0_{4}^{+}$ & 11.9 & 0.9 & - \\
$0_{5}^{+}$ & 12.6 & 1.5 & - \\
$0_{6}^{+}$ & 14.8 & 1.6 & - \\
\hline
\end{tabular}

In table 2, the calculated electric monopole (E0) matrix elements in ${ }^{18} \mathrm{O}$ are compared with the experimental data. In the $\alpha+{ }^{14} \mathrm{C}$ cluster model, the $0_{3}^{+}$state is missing because this state is considered to have the dominant configuration of ${ }^{16} \mathrm{O} \otimes v\left(1 s_{1 /}\right)^{2}$, which is difficult to describe by the $\alpha$ cluster model. The $0_{2}^{+}$state appears at the low excitation energy of $E_{x} \sim 4 \mathrm{MeV}$ but the observed E0 strength to this state is 1.5 times larger than the strength for the single particle excitation [16]. Theoretical calculation is 1.1 in a unit of the single particle strength, and this result nicely reproduces the observed strength [11].

Table 2. Same as table 1 but for ${ }^{18} \mathrm{O}$ with the E0 strength [11]. Experimental data of $\mathrm{E} 0$ and energy of $0_{4}^{+}$are taken from Refs. [16] and [17], respectively.

\begin{tabular}{llll}
\hline State & Energy & E0 (Th.) & (Exp.) \\
\hline $0_{2}^{+}$ & $4.3(3.6)$ & 1.1 & 1.5 \\
$0_{4}^{+}$ & $8.7(9.9)$ & 0.5 & - \\
$0_{5}^{+}$ & 15.6 & 1.6 & - \\
\hline
\end{tabular}

From the results shown in tables 1 and 2, we can clearly understand that the $\alpha$ cluster model is possible to 
reproduce the enhanced and discrete monopole strength at the low excitation energy below $E_{x}=15 \mathrm{MeV}$. The reproduction of the low-lying discrete strength is difficult in the naive mean field picture because the monopole excitation of the single nucleon motion requires the $2 \hbar \omega$ jump in energy, which amounts to about $30 \mathrm{MeV}$ in this mass region. For instance, in the mean field model, the monopole strength appears at much higher energy with the broad width structure [8].

Since the sophisticated shell model calculations have been done in recent studies, the comparison of such the modern shell model calculation with the $\alpha$ cluster model is very interesting. For instance, no-core shell model (NCSM) was applied to ${ }^{10,12} \mathrm{Be}$, and the energy levels in ${ }^{10} \mathrm{Be}$, which included the intruder $0_{2}^{+}$state, were beautifully reproduced [18]. The IS0 strength was not shown in the NCSM calculation but the comparison of the $\alpha$ cluster model and NCSM is very interesting in future studies.

\subsection{ISO and IS1 transition in ${ }^{44} \mathrm{Ti}$}

The macroscopic $\alpha$ cluster model is applied to the $\alpha+$ ${ }^{40} \mathrm{Ca}$ system, and we have found that the excited $0^{+}$state appears at the excitation energy of $E_{x}=12.5 \mathrm{MeV}$ with the decay width of $\Gamma=0.7 \mathrm{MeV}$. The $\alpha-{ }^{40} \mathrm{Ca}$ wave function in this excited $0^{+}$state have the one higher node in comparison to the wave function in the ground $0^{+}$state. Thus, the excitation from the ground $0^{+}$state to the excited $0^{+}$ state corresponds to the nodal excitation in the $\alpha-{ }^{40} \mathrm{Ca}$ relative motion. In the previous experiment of ${ }^{40} \mathrm{Ca}\left({ }^{6} \mathrm{Li}\right.$, d) ${ }^{44} \mathrm{Ti}$ at $E_{\alpha}=37$ and $50 \mathrm{MeV}$, the higher nodal $0^{+}$state is identified at $E_{x}=10.9 \mathrm{MeV}$ from the analysis of the differential cross section [19]. The higher nodal $0^{+}$state at $E_{x}=12.5 \mathrm{MeV}$ obtained in theory seems to correspond to the observed $0^{+}$state at $E_{x}=10.9 \mathrm{MeV}$.

The continuum strength function for the ISO transition is calculated, and we have confirmed that the prominent peak appears at the excitation energy of about $12 \mathrm{MeV}$, which corresponds to the higher nodal $0^{+}$state. Since the ISO operator strongly induces the nodal excitation in the cluster relative motion, a strong enhancement occurs in the transition from the ground state to the higher nodal state.

We calculate the ratio of the ISO matrix element of the resonant $0^{+}$state to the single particle strength. Up to the resonance energy, we sum up the total strength of the IS0 matrix element, and we have found that the total strength amounts to about 1.5 times larger than the respective single particle strength. Moreover, the enhancement factor reaches about 3 around the energy region of $E_{x}=20 \mathrm{MeV}$. In a simple mean field picture, the monopole excitation requires $2 \hbar \omega$ excitation of a nucleon motion in the $p f$ shell, which reaches about $25 \mathrm{MeV}$. In the cluster excitation, however, the IS0 strength exceeds the single particle strength at much lower excitation energy $\left(E_{x} \leq 12 \mathrm{MeV}\right)$.

We have also performed the similar calculation for the dipole $1^{-}$state. In the this calculation, two $1^{-}$resonances are obtained; the $1_{1}^{-}$state at $E_{x}=5.3 \mathrm{MeV}$ with extremely sharp width and the $1_{2}^{-}$state at $E_{x}=12.7 \mathrm{MeV}$ with $\Gamma=$ 4.4 MeV. The former state corresponds to the negative parity partner of the ground $0^{+}$state, while the latter state has the higher nodal state of the yrast $1_{1}^{-}$state at $E_{x}=5.3$ $\mathrm{MeV}$. In the ${ }^{40} \mathrm{Ca}\left({ }^{6} \mathrm{Li}, d\right){ }^{44} \mathrm{Ti}$ reaction, the $1^{-}$state is observed as the fragmented levels $\left(E_{x} \sim 6.2 \mathrm{MeV}\right.$ and 8.2 $\mathrm{MeV}$ ) with the centroid energy of $E_{x}=7.2 \mathrm{MeV}$ [19]. Since this fragmented $1^{-}$state has the large spectroscopic factor, $S_{\alpha} \sim 0.3$, the $1^{-}$state observed at the centroid of $E_{x}=7.2 \mathrm{MeV}$ seems to correspond to the $1_{1}^{-}$state at $E_{x}=$ $5.3 \mathrm{MeV}$, which is obtained by the present $\alpha+{ }^{40} \mathrm{Ca}$ cluster model, although the theoretical energy is a little lower than the experimental energy. The similar assignment was also done in previous works [19].

As for the $1_{2}^{-}$state, there is no experimental identification but a considerable mixture of the $1^{-}$strength is confirmed in the $0^{+}$resonance at $E_{x}=10.9 \mathrm{MeV}$ [19]. Since the $0^{+}\left(E_{x}=10.9 \mathrm{MeV}\right.$ and $\left.\Gamma=0.7 \mathrm{MeV}\right)$ and $1_{2}^{-}\left(E_{x}=\right.$ 12.7 MeV and $\Gamma=4.4$ ) resonances in the calculation overlap each other in energy, the considerable mixture of the $0^{+}$and $1^{-}$strengths in the experiment is consistent to the theoretical calculation.

In the calculation of the continuum IS1 strength, the strong and sharp enhancement is obtained at the resonance position of $E_{x} \sim 5 \mathrm{MeV}$. The magnitude of the IS1 matrix element from the ground $0^{+}$state to the $1_{1}^{-}$resonant state is about 4.5 times larger than the single particle strength. In the simple mean field picture, the excitation to the $1^{-}$state requires the $1 \hbar \omega$ excitation, which corresponds to about $12 \mathrm{MeV}$. In the case of the $\alpha-{ }^{40} \mathrm{Ca}$ relative excitation, the enhanced strength occurs at a half of the energy in the single particle excitation, $E_{x} \sim 5 \mathrm{MeV}$. This is the similar feature to the ISO transition. The contribution from $1_{2}^{-}$at $E_{x} \sim 15 \mathrm{MeV}$ further increases the total strength of the matrix elements, and the ratio of the total strength to the single particle strength finally reaches about a factor of about 6 in the energy region up to $15 \mathrm{MeV}$. The theoretical calculation predicts the strong enhancement in the IS1 strength and hence, the experimental measurements are strongly desired in future studies.

\subsection{IS1strength in ${ }^{104--110} \mathrm{Te}$}

We have applied the macroscopic $\alpha$ cluster model to even Te isotopes, such as ${ }^{104-110}$ Te with $\alpha+{ }^{100-106} \mathrm{Sn}$. The strength of the DF potential is slightly tuned (1.03) so as to reproduce the experimental $Q$-value of the $\alpha$ decays in ${ }^{104-110} \mathrm{Te}$ isotopes. The continuum strength function for the IS1 transition are calculated by employing the renormalized DF potentials.

In all of the Te isotopes, we have confirmed that the sharp and broad peaks appear in the IS1 strength around the excitation energy of $E_{x} \sim 5 \mathrm{MeV}$ and $E_{x} \sim 13 \mathrm{MeV}$, respectively. The IS1 matrix elements for the $E_{x}=5 \mathrm{MeV}$ states are comparable to the single particle unit, and the strength in ${ }^{104} \mathrm{Te}$ is the largest of all isotopes. The IS1 matrix element in ${ }^{104} \mathrm{Te}$ is about 0.9 in a unit of the single particle strength. Since $1 \hbar \omega$ for the single particle excitation is about $9 \mathrm{MeV}$ in this mass region, we can easily understand that the dipole excitation of the $\alpha$ particle occurs at much lower excitation energy $\left(E_{x} \sim 5 \mathrm{MeV}\right)$ in comparison to the single particle excitation. 
In even Te isotopes, the experimental observations of the low-lying electric dipole (E1) strength were reported in the mass region around the $\beta$ stability line, ${ }^{122-130} \mathrm{Te}$ [20]. Some enhanced structures were observed in $E_{x} \leq$ $5 \mathrm{MeV}$, which were analyzed by two phonon model [20] and quasi-particle random phase approximation with deformed single particle basis [21]. It is very interesting to extend the measurement of the low-lying E1 strength to the lighter Te isotopes, ${ }^{104-110} \mathrm{Te}$, while the application of $\alpha$ cluster model to the heavier Te isotope is important subject in future work.

\section{4 summary and discussion}

In summary, we have applied the microscopic and semimicroscopic $\alpha$ cluster models to the lighter systems, such as ${ }^{12} \mathrm{Be}$ and ${ }^{18} \mathrm{O}$, and clearly demonstrated that the prominent enhancements occurs in the isoscalar or electric monopole matrix elements going to the low-lying discrete $0^{+}$levels. This enhancement is induced by the excitation in the relative motion between the $\alpha$ cluster and the residual nucleus. Nevertheless the strength of the monopole transition is comparable to or exceeds the single particle strength, the excitation energy of such the $\alpha$ monopole excitation is much lower than the energy of the single nucleon excitation in the mean field picture. Therefore, the low-lying and discrete strength in the monopole transition is characteristic phenomena in the $\alpha$ cluster structure.

We have also extended the analysis about the isoscalar monopole (IS0) transition to the heavier system of ${ }^{44} \mathrm{Ti}$, in which the macroscopic cluster model with $\alpha+{ }^{40} \mathrm{Ca}$ known to work successfully. The continuum strength of the IS0 transition is calculated, and we have found that the strong IS0 transition, which exceeds the respective single particle strength, appear at the low excitation energy region, which is about a half of $2 \hbar \omega$ for the single nucleon excitation. Thus, the low-lying enhancement in the monopole matrix element has also been confirmed in the heavy system.

In addition, we have also calculated the continuum strength of the isoscalar dipole (IS1) transition for the $\alpha$ $+{ }^{40} \mathrm{Ca}$ system, which has been proposed as a effective probe for the asymmetric cluster system [9]. The matrix element of the IS1 transition is prominently increased in the low excitation region, and this result is similar to the result in the ISO transition. Furthermore, the calculations of the continuum IS1 strength is also performed for ${ }^{104-110} \mathrm{Te}$ isotopes by applying the $\alpha+{ }^{100-106} \mathrm{Sn}$ cluster model. The calculation of Te isotopes has also supported the low-lying enhancement of the IS strength generated by the $\alpha$ cluster excitation.

From the results of a series of our calculations, the low-lying enhancement in the monopole and dipole transitions, which is induced by the $\alpha$ cluster excitation, seems to generally occur over a wide range of nuclear systems. The excitation in the relative motion of the $\alpha$ cluster and the residual nucleus can naturally describe the low-lying enhancement in the strength. In the analysis of the heavy systems, however, we must consider the breaking effect of $\alpha$ cluster generated by coupling to the mean field configuration more explicitly. In particular, the coupling of the $\alpha$ configuration and the shell model configuration is quite important in the initial ground state in the heavy systems. This coupling will reduce the total strength of the monopole and dipole transition. Moreover, the decays of the final $\alpha$ cluster state into the more complicated compound states will be important because the excited monopole and dipole states are embedded in continuum. This decay effect will spread the discrete IS strength.

The revised theoretical calculation to take into account the breaking and spreading effects on the $\alpha$ cluster state are now underway.

\section{References}

[1] P. Ring and P. Schuck, 2004 Nuclear Many-Body Problem1st edn (Berlin: Springer), and references therein.

[2] H. Horiuchi et al., Suppl. Prog. Theor. Phys. 192, 1 (2012), and references therein.

[3] U. Atzrott, P. Mohr, H. Abele, C. Hillenmayer, and G. Stadt, Phys. Rev. C53, 1336 (1996).

[4] F. Michel, S. Ohkubo and G. Reidmeister, Suppl. Prog. Theor. Phys. bf132, 7 (1998), and references therein.

[5] M. Kimura and H. Horiuchi, Nucl. Phys. A767, 58 (2006).

[6] M. A. Souza and H. Miyake, Phys. Rev. C91, 034320 (2015), and references therein.

[7] Y. Suzuki and S. Ohkubo, Phys. Rev. C82, 041303(R) (2010), and references therein.

[8] T. Yamada, Y. Funaki, T. Myo, H. Horiuchi, K. Ikeda, G Röpke, PŚchuck, and A. Thosaki, Phys. Rev. C85, 034315 (2012).

[9] Y. Chiba, Y. Taniguchi and M. Kimura, Phys. Rev. C95, 044328 (2017).

[10] M. Ito and K. Ikeda, Rep. Prog. Phys. 77096301 , (2014).

[11] M. Nakao, H. Umehara, S. Ebata and M. Ito, Phys. Rev. C98, 054318 (2018).

[12] M. E. Brandan and G. R. Satchler, Nucl. Phys. A487, 477 (1988).

[13] S. Ebata, T. Nakatsukasa, T. Inakura, K. Yoshida, Y. Hashimoto, and K. Yabana, Phys. Rev. C82, 034306 (2010).

[14] M. Iwasaki, R. Otani, Y. Takenaka and M. Ito, PTEP 2015, 023D01, and references therein.

[15] Z. H. Yand et al., Phys. Rev. C91 024304 (2015).

[16] K. H. Souw et al., Phys. Rev. C11, 1899 (1975).

[17] M. L. Avila et al., Phys. Rev. C90, 024327 (2014).

[18] L. Liu et al., Phys. Rev. Cbf96, 014302 (2012).

[19] T. Yamaya, K. Katori, M. Fujiwara, S. Kato and S. Ohkubo, Suppl. Prog. Theor. Phys. 132, 73 (1998), and references therein.

[20] R. Schwengner et al., Nucl. Phys. A620, 277 (1997).

[21] E. Guliyev et al., Phys. Lett. B532, 173 (202). 